\title{
THE CLINICAL APPLICATION OF THE COMPLEMENT FIXATION TEST FOR GONORRHOA
}

By I. N. ORPWOOD PRICE, Pathologist, Whitechapel Clinic.

THIs paper has been written in the hope that it will be of help to those clinicians who may have occasion to use the complement fixation test for gonorrhœa in the diagnosis of disease.

No details of the technical performance of this test are given, and those readers who are interested in the new technique which I have elaborated are referred to the monograph ${ }^{1}$ which I have written on this subject.

\section{Clinical Application}

In applying the results given by this new method of performing the complement fixation test for gonorrhœa to the clinical condition of the patient at the time of the test, it is essential to have some idea of the immunological factors at work.

It must be clearly understood that the test is a serum reaction which registers the presence or absence of specific gonococcal antibodies in the blood; the reaction itself does not signify the presence or otherwise of gonococci in the tissues. In general terms it may be stated that the production of antibodies is part of an infection, and that the amount of antibody so produced varies directly with the amount of toxin absorbed together with the intensity of the reaction of the tissues against that toxin. The quantity of toxin absorbed depends on the amount produced by the infection and whether the infection is " closed " or " open." If the infection is "open" and if good drainage of the toxic products from the area of infection is established, little or no toxic absorption occurs and then the antibody response remains in abeyance. In a "closed" case where the toxic products are unable to drain efficiently, toxic absorption and the production of antibodies usually proceed apace. Exceptionally, cases occur in which, in 


\section{BRITISH JOURNAL OF VENEREAL DISEASES}

spite of an apparently " closed " infection, the tissues do not seem to react to the presence of toxin and little antibody response is evoked.

In applying these facts to gonorrhœa, it must be remembered that this disease is primarily an epithelial or "surface" infection and that the genital tract, owing to its anatomical peculiarities, affords numerous opportunities for such an infection to become " closed." For example, if during an attack of gonorrhœa the seminal vesicles become involved and, as is quite common, are apparently occluded from the rest of the genital tract, the conditions of a "closed" gonococcal infection immediately exist.

Therefore it may be stated that a positive complement fixation reaction is indicative of the presence of gonococcal antibodies formed in the blood-serum as a result of absorption of the toxic products from a gonococcal infection. It follows from this that such a patient must be regarded as infectious.

Conversely, a negative reaction indicates the absence of gonococcal antibodies in the blood-serum, but this does not exclude the presence of a gonococcal infection. Thus, whilst a negative reaction will naturally be recorded in the absence of a gonococcal infection, under certain conditions a negative result may be recorded even when gonococci are present in the tissues. Since this type of reaction is apt to cause confusion in the minds of some clinicians, the conditions which cause it will be considered in detail. They may be divided into three categories :-

(i) Early Acute Gonorrhoea.-In the early acute stage of the disease when the infection is limited to the anterior urethra in the male, or to "blenorrhagie basse" in the female, although gonococci may be demonstrated in the discharges, the complement fixation test is negative. This lack of antibody in the blood serum of these patients, as shown by the negative complement fixation test, is probably due to two reasons: (a) a latent period is required for the elaboration of antibody; and $(b)$ these types of infection are at first "open" or " surface" infections with good drainage and little, if any, of the toxic material is absorbed; hence the body is insufficiently stimulated to produce antibodies.

(ii) Treated Chronic Gonorrhoea.-This class is more important. It includes, for example, the patient who has 


\section{FIXATION TEST FOR GONORRHCA}

been treated by digital massage for a chronic gonococcal vesiculitis for some time. Clinically and serologically he gives negative results, but smears made from the vesiculoprostatic contents reveal on careful searching the presence of gonococci. This may be confirmed by cultures. Upon investigating 200 such patients, I discovered that gonococci could be demonstrated in the vesiculo-prostatic smears in 3 per cent. of them, whilst the complement fixation test was negative. The interpretation of this phenomenon I believe to be as follows: An untreated chronic gonococcal vesiculitis is, in the majority of cases, one in which drainage is either absent or inadequate, and as such records a positive serum test. On treatment by digital massage, conditions of an "open" infection obtain when drainage is established, and usually the serum test rapidly becomes negative although gonococci may be still present in the tissues. Therefore the possibility of this sequence of events must be kept in mind particularly when a " test of cure" is undertaken.

(iii) Failure of the Tissues to respond to the Presence of Toxin.-Rarely (O.I per cent. of cases) it would appear that the tissues fail to respond to the presence of a gonococcal infection even although conditions of a "closed" infection seem to exist. In this type of case it is difficult to be certain that the infection is " closed," but in any event the complement fixation test remains negative throughout the course of the disease. Fortunately, the gonococcus can usually, but not always, be isolated with comparative ease.

Therefore, the type of reaction to be expected at the time of the test can be surmised by considering the clinical condition of the patient. If the infection is an " open " one with good drainage, a negative result may be anticipated; if partial drainage is established, a positive $(+)$ result is to be expected ; whilst if the infection is " closed" a strongly positive $(++)$ result should be obtained.

From the above brief survey of the clinical application of the test it is obvious that in order to interpret the complement fixation test for gonorrhœa a knowledge of the clinical history and condition of the patient, together with a rational idea as to the condition under which specific antibody is produced, is essential.

The production of specific gonococcal antibody depends 


\section{BRITISH JOURNAL OF VENEREAL DISEASES}

on: (i.) the age of the infection ; (ii.) the anatomical spread of the disease ; (iii.) the reaction of the tissues to the presence of gonococci ; and (iv.) the stage of infection at which treatment is instituted, and the degree of its therapeutic deficiency.

(i.) The Age of the Infection.-To estimate the stage of the infection at which a positive reaction might be expected, the following graph was constructed from the results of tests performed for the first time on sera from untreated cases of gonorrhœa in which the age of the infection was accurately known.

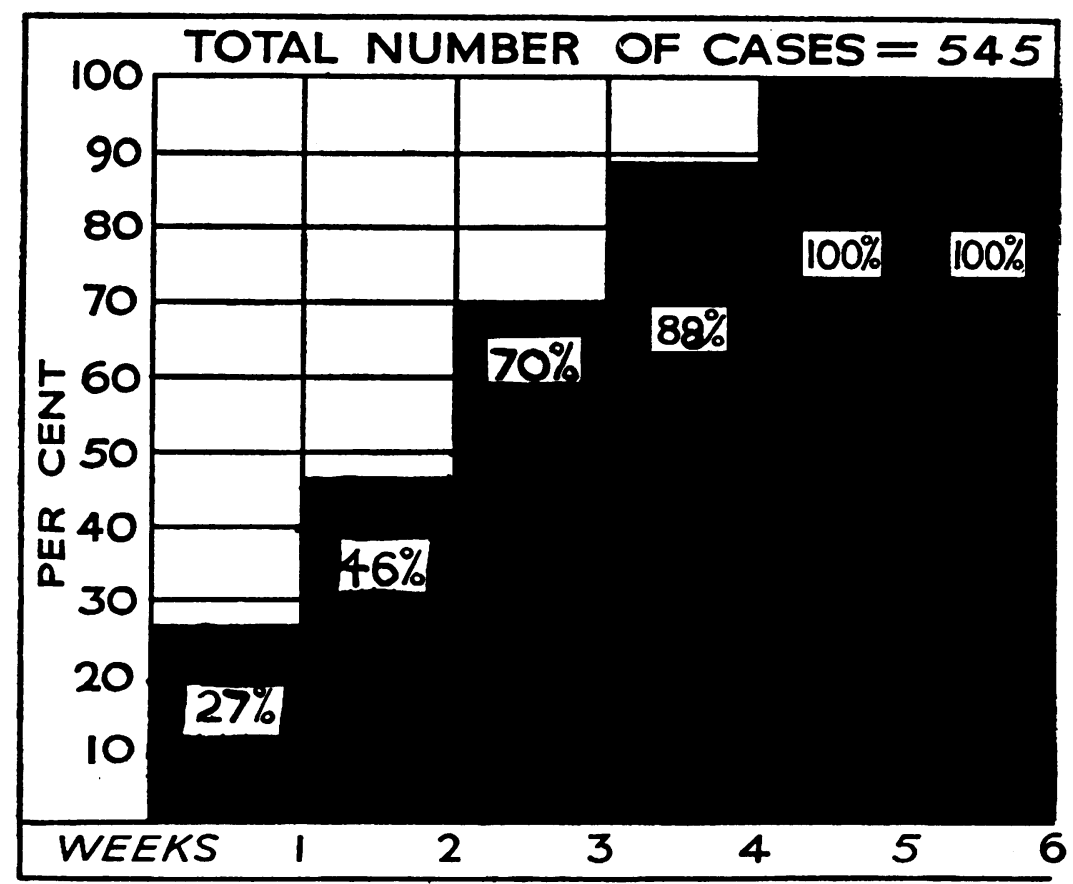

FIG. I.

This diagram shows that the likelihood of obtaining a positive result varies within limits, directly with the age of the infection. In the first week after exposure to infection 27 per cent. (69 in a total of $25 \mathrm{I}$ ) of positive reactions were given. A rise to 46 per cent. (82 out of I80) of positive reactions occurred in the second week. In the third week this rise continues and 70 per cent. (47 out of 67 ) positives were recorded. In the fourth week the figure obtained was 88 per cent. (2I from a total of 24). In the fifth and sixth weeks Ioo per cent. positive 


\section{FIXATION TEST FOR GONORRHEA}

results were recorded, the figures being 23 out of 23 . From these figures one fact emerges : viz., the longer the disease is allowed to go unchecked the greater becomes the absorption of toxin and also the greater is the likelihood of a " closed " infection supervening with a resultant positive reaction.

(ii.) The Anatomical Spread of the Disease.-This depends on the age of the infection, the reaction of the tissues, and the treatment, if any, adopted.

In the male, case records show definitely that if the infection is limited to the anterior urethra a negative, or at most a weakly positive reaction $( \pm)$ is recorded, unless some of the deeper structures such as Littré's follicles or Cowper's glands have become involved, in which case a positive reaction $(+)$ is the rule. When the disease has spread to the posterior urethra a strongly positive $(+ \pm)$ or $(++)$ reaction quickly results. For some years I have noticed the rapidity and frequency with which a negative reaction changes to positive when the posterior urethra becomes involved. Possibly this is due to the great vascularity of the posterior urethra, and to the high percentage of vesicular infections which occur as soon as the posterior urethra becomes infected. Recently, research work undertaken in conjunction with my medical colleagues at this clinic suggests how frequently a gonococcal vesiculitis is associated with posterior urethritis in the acute stage of the disease. In many cases when infection spreads to the posterior urethra, it would appear that the seminal vesicles become almost simultaneously involved. This leads in most cases to conditions of a "closed " infection, and owing to the excellent blood supply to these organs absorption of toxic material is rapid and the consequent early appearance of antibody in the blood stream results in the speedy production of a positive serum reaction.

In the female, a gonococcal urethritis alone rarely causes a positive reaction, but a gonococcal Bartholinitis usually results in a positive $(+)$ serum reaction. When, however, the infection spreads to the uterus and the Fallopian tubes, a strongly positive reaction $(+ \pm$ or $++)$ is the rule.

(iii.) The Reaction of the Tissues.-In I93I I stated that tissues seem to respond somewhat slowly to the presence of gonococci, although antibody production commences during the first week of the infection. Since 


\section{BRITISH JOURṆAL OF VENEREAL DISEASES}

then, owing to the results obtained through the increased sensitivity of the new technique that view must be modified, and I have now found that once absorption of toxin occurs, with its resulting formation of antibody, a positive complement fixation test is recorded in as short a time as 3 to 4 days. For example, I have produced in myself and in rabbits a positive $(+)$ reaction in the short space of 4 days by the daily injection of vaccine. By the tenth day a strongly positive $(++)$ reaction was obtained. Vaccine was injected subcutaneously into myself and intravenously into rabbits daily for 9 successive days in doses beginning at 0.25 c.c. and working up to $2 \cdot 0$ c.c. One c.c. of vaccine was equivalent to 360 millions of organisms. It is interesting that the positive reaction became negative six weeks after the cessation of the vaccine administration. In collaboration with A. J. King I have given the same vaccine subcutaneously in similar doses to patients who were suffering from acute gonorrhœa of 4 or 5 days' standing. Serum tests were performed at the outset, after 3 days, 6 days and finally after ro days. A typical result is shown graphically below :-

Strength of C.D.T.

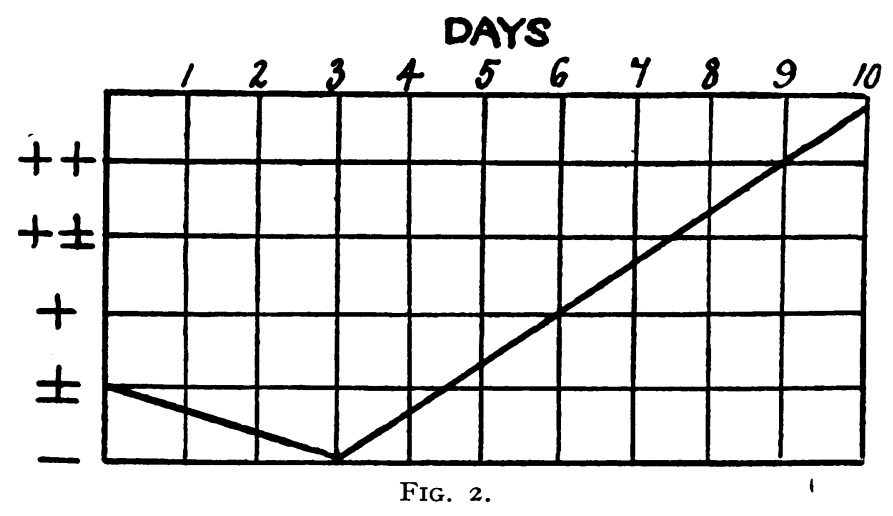

The serum on the first testing recorded a weakly positive $(+)$ reaction. By the third day this had become negative, which may be taken as the "negative phase." On the sixth day the serum had become positive $(+)$ again, and by the ninth day the result had increased to a strongly positive $(++)$ reaction. So that the latent period of antibody production is about 3 to 4 days; and 


\section{FIXATION TEST FOR GONORRHEA}

if conditions are such as to ensure the absorption of toxin into the system, antibody makes its appearance comparatively rapidly in the blood stream.

(iv.) The Stage of the Infection at which Treatment is Instituted.-(a) Acute gonorrhœa : If adequate treatment is adopted early in the disease, that is, during the first week after infection, the reaction may only become weakly positive $( \pm)$, or it may even remain negative throughout the course of the infection. These cases are usually rapidly cured, and lack of antibody in the blood stream (as shown by the negative serum test) is possibly due to the localisation of the disease to the anterior portion of the urethra in the male, or in the case of the female to the lower and "surface" tissues of the genitalia with no involvement of the deeper structures, such as Bartholin's glands.

(b) Sub-acute gonorrhœa: If the case is not seen until three or four weeks after exposure to infection, a positive reaction is generally recorded. In the male, it will often be found that the seminal vesicles have become infected and that gonococci can be demonstrated in the vesiculoprostatic fluid by smears and cultures. In the female, it is often difficult to obtain positive smears and cultures in these sub-acute cases even when gonococci are almost certainly present, as for example in " contact" cases, and then the simplest and most satisfactory means at our disposal to confirm the clinical picture is the complement fixation test for gonorrhœa.

(c) Chronic gonorrhœa: One is frequently confronted with male cases which have passed into the chronic stage of the disease because they defaulted when being treated in the earlier stages of the infection. They frequently have little in the way of signs or symptoms, and the problem arises as to whether they are free from disease. One of the surest methods of deciding this is by the result of the complement fixation test. If this is positive the patient must be regarded as infectious, and a diligent search must be made for the focal site. The seminal vesicles are often at fault. Clinical examination of these organs may demonstrate that they are pathological, and gonococci may be found in the vesicular fluid by means of smears and cultures. Efficient treatment, such as vesicular massage, will promote drainage from such a focus of infection and the complement fixation test will become 


\section{BRITISH JOURNAL OF VENEREAL DISEASES}

negative. It must be clearly realised that, if a positive serum reaction is reduced to negative after a course of massage, this is no guarantee that all gonococci have been removed; and, in such a case, one negative serum result must not be taken as indicating that the patient is cured. It does, however, show that drainage has been established, and that absorption of toxin has ceased. If massage is prematurely stopped, the serum test may later again become positive. This shows that active gonococci still exist and that absorption of toxin has recommenced. This is of the greatest importance in considering the value of the complement fixation reaction as a test of cure. I may have unduly stressed the case of the gonococcal vesicle in chronic gonorrhœa, but I suggest that herein lies most of our trouble in the treatment of chronic gonorrhœa in the male. In many cases the seminal vesicles act unsuspectedly as a reservoir of infection and intermittently infect the rest of the genital tract. If these organs when infected are adequately treated, many of the other manifestations of chronic gonorrhœa rapidly subside. Serologically these tests run parallel with the efficacy of the treatment up to the point where adequate drainage is established and absorption of toxin has ceased. Thereafter, the test will remain negative as long as efficient drainage is maintained or if the disease has been eradicated. I would repeat that if the disease is not cured, and if treatment has been stopped prematurely because of the negative serum test, then the complement fixation test will again become positive.

The complement fixation test is nearly always positive in cases of chronic gonorrhœa in the female, since the infection in these cases frequently involves the uterine cavity and its adnexa, which from their anatomical arrangement tend to form a "closed" focus of infection. These organs are very vascular, the difficulty of draining the uterus efficiently is well known, and therefore toxic absorption is favoured. Successful treatment in these cases is indicated by a falling off of the strength of the serum reaction, and until a positive reaction is reduced to a negative the patient must be regarded as infectious. When this occurs the patient is generally cured, although a cervicitis due to other organisms such as staphylococci or the colon bacillus, may still persist.

Gonococcal Complications.-Serologically as well as 256 


\section{FIXATION TEST FOR GONORRHEA}

clinically gonococcal complications may be divided into two groups, acute and chronic.

Acute complications usually supervene when the serum test is already positive, and always result in it becoming more strongly positive $(++)$. Thus, an acute epididymitis or salpingitis may cause so much toxic absorption and tissue reaction that a strongly positive complement fixation test is recorded even when the serum is diluted to $\mathrm{I} / 8 \mathrm{o}$ (neat serum is used in the standard test).

Chronic complications such as a chronic gonococcal joint, tenosynovitis, or chronic gonococcal rheumatism usually record a positive $(+)$ reaction.

The more frequent use of the complement fixation test for gonorrhœa would lead to a higher percentage of correct diagnoses of many " rheumatic " cases of doubtful origin.

\section{VACCINES}

The effect of vaccines on the strength of the complement fixation reaction largely depends on the type of vaccine used as well as on the tissue reaction of each particular patient. It is frequently stated that the administration of vaccine may nullify the value of the complement fixation test for gonorrhœa. After careful study and experiment I have come to the conclusion that this is not so. As a result of injecting vaccine one of two things may happen. Either

(i.) the vaccine may have no effect on the disease and may fail completely to stir up the infection (in this case the strength of the serum reaction may show a temporary rise, but in my experience this returns to its original value within six weeks after the last injection); or

(ii.) the vaccine may cause a "flare-up" at the focus of infection and in effect cause a chronic manifestation of the disease to become acute. The result serologically is a strongly positive $(++)$ reaction, and this is due partly to the reaction of the tissues against the vaccine, but it is chiefly the result of the absorption of toxin elaborated by gonococci which have been stimulated into action by the vaccine. As stated above, the vaccine reaction ceases to show serologically about six weeks after the last injection. So 


\section{BRITISH JOURNAL OF VENEREAL DISEASES}

that if the strength of the complement fixation test remains at a higher level than it was before the administration of the vaccine, this must be due to the absorption of toxin of the causative organisms of the disease. Therefore, if vaccine has been given during the course of the disease, any subsequent positive serum reaction is not of necessity due to the vaccine.

\section{Vulvo-vaginitis of ChILdREN}

At present I have had little opportunity of testing the value of this new technique in cases of gonococcal vulvovaginitis of children; I am able to survey the results on only 35 patients. Of these I 8 were diagnosed clinically as suffering from a non-gonococcal vulvo-vaginitis, and in spite of repeated tests by smears and cultures, this diagnosis was confirmed as gonococci were never demonstrated. In these cases the serum tests remained negative throughout the course of the illness. Three cases in which the gonococcus was isolated were tested once only during the first week of the infection; these gave negative serum results. The remaining I4 cases had positive reactions and exhibited gonococci in the secretions. From these results and taking into consideration a somewhat larger series of cases tested by the old technique, it would appear that results similar to those obtained in the adult are to be expected in children.

\section{CRoss-FiXation}

The question of cross-fixation with the antibodies of allied organisms such as the micrococcus catarrhalis, the meningococcus and the micrococcus flavus (particularly type 2) is sometimes raised. It undoubtedly becomes of importance when a patient's serum gives a positive complement fixation test for gonorrhœa in the absence of clinical or other pathological evidence in support of this disease, whilst definite or indefinite catarrhal symptoms of the nose and throat may be present. The possibility of this phenomenon has often been discussed, but usually with inconclusive results. As long ago as I9I2, Arkwright, ${ }^{2}$ after many experiments, concluded that crossfixation with antibodies of the meningococcus might occur when some strains of gonococci were used as an antigen. Oliver ${ }^{3}$ (using Thomson's antigen and tech258 


\section{FIXATION TEST FOR GONORRHEA}

nique) came to the conclusion that in rabbits under experimental conditions, a considerable degree of crossfixation between the antiserum of $\mathrm{m}$. catarrhalis and gonococcal antigen may occur. Using my old technique, $\mathrm{I}^{4}$ found only two cases among 7,000 in which the possibility of cross-fixation by the antibodies of $\mathrm{m}$. catarrhalis could not be excluded, and I suggested a method of determining such cases by means of monthly parallel quantitative tests using gonococcal and catarrhalis antigens. Mascall, ${ }^{5}$ in I93I, reported a case of a child in which, as the result of bacteriological and serological investigations, he came to the conclusion that the patient (who had a persistently positive gonococcal fixation test, in the absence of any clinical or other pathological evidence of gonococcal infection) was suffering from a flavus (type I) infection of the nose and throat, and that the positive gonococcal fixation test was due to crossfixation with the antibodies of $\mathrm{m}$. flavus (type $\mathrm{I}$ ).

Therefore when this new method of performing the gonococcal fixation test was evolved, it became important to ascertain whether such cross-fixation did occur when using the new antigen, and if so, to what extent. I have been able to collect a few clinical cases and some evidence which is mainly experimental, but some years must elapse before sufficient knowledge can be obtained by which the significance of this phenomenon can be accurately estimated from the clinical point of view.

\section{EXPERIMENTAL WORK}

Antigens of the gonococcus, m. catarrhalis, the meningococcus and $\mathrm{m}$. flavus were treated separately against the antiserums respectively. The antisera were obtained by injecting rabbits and guinea pigs with the respective organisms. The antigens of the meningococcus and $\mathrm{m}$. flavus were prepared in a similar manner to the new standard preparation of gonococcal antigen. The antigen of the micrococcus catarrhalis was made in a similar manner except that antiformin was used as a solvent for the organism instead of $\mathrm{N} / \mathrm{NaOH}$ as in the case of the gonococcus. All the experiments were performed in an identical manner, but for the sake of clearness one of these will be described in detail.

Serial dilutions of gonococcal antigen ranging from 


\section{BRITISH JOURNAL OF VENEREAL DISEASES}

I-Io to I-8o were mixed with equal volumes of gonococcal, catarrhalis, meningococcal and flavus antisera, and to each mixture an equal volume of 3 m.h.d. complement was added. After incubation at $37.5^{\circ} \mathrm{C}$. for one hour, an equal volume of sensitised red cells was added; and then, after a further thirty minutes' incubation at the same temperature, the amount of complement fixation obtained by each mixture was noted. The results can be diagramatically represented :-

Serum controls.

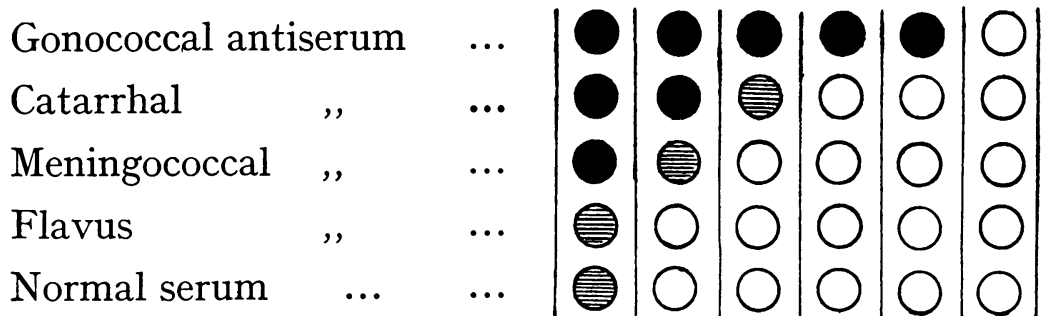

Gonococcal antigen dilutions I/IO I/20 I/40 I/60 I/80

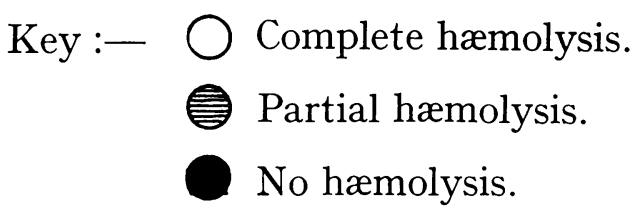

FIG. 3.

The above diagram shows that complete fixation is obtained by gonococcal antiserum even when gonococcal antigen is used in a dilution of $\mathrm{r} / 8 \mathrm{o}$ (actually the endpoint was determined at a dilution of I/I30), whilst with a catarrhalis antiserum complete fixation occurred when the gonococcal antigen was diluted to approximately $\mathrm{I} / 30$. The meningococcal antiserum gave complete fixation when the gonococcal antigen was diluted approximately to I/I 5 ; whilst, if the latter were used at a strength of I/Io only, incomplete fixation was given by the flavus antiserum. No fixation occurred with a normal serum at antigen dilution of approximately I/I 5 , whilst the serum controls proved to be free from anti-complementary action. It should be noted that both the flavus and meningococcal antisera and antigens employed were of a polyvalent type. 


\section{FIXATION TEST FOR GONORRHEA}

Since the titre of such a gonococcal antigen would be taken as I in 30 (i.e., half that amount of antigen which just failed to fix $3 \mathrm{~m}$.h.d. of complement in the presence of a normal serum), it was concluded that in routine tests the possibility of cross-fixation with antibodies of the meningococcus or $\mathrm{m}$. flavus could be ignored. It is evident, however, that a weak degree of cross-fixation with the antibodies of $\mathrm{m}$. catarrhalis does occur under experimental conditions. In fact, when such an antiserum as that used in the above experiment is tested by the standard gonococcal complement fixation test, a weakly positive $( \pm)$ reaction is recorded. But it would require a very heavy infection with $\mathrm{m}$. catarrhalis for the serum of a patient to give such a reaction $( \pm)$, and in view of the symptoms that such an infection would occasion, it seems doubtful if the test would place the diagnosis in jeopardy.

Further experiments on similar lines were conducted by titrating serial dilutions of catarrhalis, meningococcal and flavus antigens against gonococcal, catarrhalis, meningococcal and flavus antisera respectively.

The end points of these titrations are shown in the table below :-

\begin{tabular}{|c|c|c|c|c|}
\hline \multirow{2}{*}{ Antisera. } & \multicolumn{4}{|c|}{ Complete fixation given by the highest dilution antigen used. } \\
\hline & $\begin{array}{c}\text { Gonococcal } \\
\text { titre } 1 / 30 .\end{array}$ & $\begin{array}{l}\text { Catarrhalis } \\
\text { titre } 1 / 35 .\end{array}$ & $\begin{array}{l}\text { Meningococcal } \\
\text { titre } 1 / 25\end{array}$ & $\begin{array}{c}\text { Flavus } \\
\text { titre } \mathrm{I} / 20 .\end{array}$ \\
\hline Gonococcal . & I $/ 130$ & $1 / 30$ & $\mathrm{I} / 20$ & $\begin{array}{c}\text { Incomplete at } \\
\text { I/IO }\end{array}$ \\
\hline Meningococcal & I $/ 15$ & $\begin{array}{c}\text { Incomplete at } \\
\text { I/10 }\end{array}$ & $I / 60$ & $\begin{array}{c}\text { Incomplete at } \\
\text { I/IO }\end{array}$ \\
\hline Catarrhalis . & $I / 30$ & $\mathrm{I} / 70$ & I/IO & $1 / 10$ \\
\hline Flavus & I/IO & $\begin{array}{c}\text { Incomplete at } \\
\text { I/IO }\end{array}$ & I/IO & $I / 50$ \\
\hline Normal serum & $\begin{array}{c}\text { Incomplete at } \\
\text { I/IO }\end{array}$ & $\begin{array}{c}\text { Incomplete at } \\
\text { I/10 }\end{array}$ & $\begin{array}{c}\text { Incomplete at } \\
\text { I/IO }\end{array}$ & $\begin{array}{c}\text { Incomplete at } \\
\text { I/10 }\end{array}$ \\
\hline
\end{tabular}

These experiments show that if the antigens are used at their respective titres as previously determined (see top of the table) the only possibility of a cross-fixation reaction occurring during the performance of the complement fixation test for gonorrhœa would be when a very strong catarrhalis antiserum was tested. In actual practice and in spite of repeated investigations, I have yet to meet a case in which the occurrence of a positive 


\section{BRITISH JOURNAL OF VENEREAL DISEASES}

gonococcal fixation test as performed by this new technique has been traced to a catarrhalis infection.

\section{Clinical Evidence}

The evidence which I have been able to collect clinically is meagre but instructive. Two types of cases are represented.

(i.) Catarrhalis Infection.-A female child (aged nine months) had suffered from snuffles since birth, and as the mother had been treated for gonorrhœa one year previously, it was thought that the child's complaint might be due to a nasal gonococcal infection. Gram-negative diplococci were found in the nasal discharge. Morphologically and culturally these were identical with micrococcus catarrhalis. In the meantime the child's serum which had been tested by the complement deviation reaction recorded a negative result with a gonococcal antigen, and a strongly positive $(++)$ result with a catarrhalis antigen. Thus the causal organism was demonstrated serologically with the complete absence of any cross-fixation.

(ii.) Meningococcal Infection.-Through the courtesy of Dr. Coyle, of St. Peter's Hospital, who supplied me with specimens, I was able to examine the blood and cerebrospinal fluid from six children who were suffering from cerebro-spinal meningitis. In each case the duration of the disease was a week or more and they had all been treated with antimeningococcal serum. The blood sera of all six cases were negative when tested by the complement fixation test for gonorrhœa. The same test when applied to the cerebro-spinal fluids gave five negative results, whilst the sixth had a weakly positive ( \pm ) reaction. Unfortunately, at this time a meningococcal antigen was not available, and so I was unable to test these specimens by a meningococcal complement fixation reaction. This would have afforded an interesting comparison, but even so, it would appear from the results obtained that cross-fixation with antibodies of the meningococcus during a meningococcal infection is very unlikely to occur.

\section{CONCLUSIONS}

By using the new technique and the new antigen for the performance of the complement fixation test for gonor262 


\section{FIXATION TEST FOR GONORRHEA}

rhœa, the probability of cross-fixation reactions occurring with the antibodies of other gram-negative cocci is excluded, with the possible exception of the micrococcus catarrhalis. That such a cross-fixation reaction with the antibodies of $\mathrm{m}$. catarrhalis should occur is so rare that, coupled with the weakness of this reaction, it is very doubtful if the result of such a test would confuse the diagnosis. Should any doubt arise, it can be dealt with by performing parallel complement fixation tests using gonococcal and $\mathrm{m}$. catarrhalis antigens and comparing the relative strengths of their reactions.

\section{INTERPRETATION OF RESULTS}

The interpretation of results must be correlated with the clinical history and the condition of the patient if a reliable opinion is to be given on the result of a serum test. It is desirable to have some idea of the "serological course " of the disease. Whilst it is obvious that the responsibility for the necessity of a complement fixation test at any given time during the course of the dieases must rest with the clinician, if full advantage is to be taken of the reaction it is advisable to have an orderly sequence of serum tests. The following scheme is suggested for use in venereal clinics: The patient's serum should be tested on the first appearance at the clinic. Whether the result of this be negative or positive, it should be repeated a month later, and thereafter at two monthly intervals until such time as clinically a test of cure is indicated. Further tests depend on all the evidence available : clinical, pathological and serological. In this way a curve of the serum reactions may be plotted which gives a graphic picture of the serological course of the disease and indicates to a large extent the effect of the treatment adopted.

The notation used is as follows :-

$$
\begin{aligned}
& ++ \text { very strongly positive } \\
& + \pm \text { strongly positive } \\
& + \pm \text { positive } \\
& \pm \text { weakly positive } \\
& \pm \text { negative }
\end{aligned}
$$

$A$ strongly positive result $(++$ or + \pm ) is definite evidence that the patient is suffering from gonorrhoea and must be regarded as infectious. In the male, it may 


\section{BRITISH JOURNAL OF VENEREAL DISEASES}

be taken that a posterior urethritis and its possible complications, and in the female that the cervical canal and higher adnexa, are involved. Such a result indicates that the infection is active and that toxic products are being absorbed into the blood stream in relatively large quantities. Acute gonococcal complications are usually accompanied by a strongly positive serum test.

$A$ positive reaction $(+)$ is definite evidence that the patient is suffering from gonorrhœa; this may occur in the early or in the chronic stage of the disease. It is usually an indication that the posterior urethra in the male, or the upper part of the cervical canal in the female, is infected ; but such a reaction may result from gonococcal Bartholinitis in the female, or Cowperitis in the male.

$A$ weakly positive reaction $( \pm)$ is obtained in the following circumstances :-

(i.) An early case, i.e., one to three weeks after exposure to infection. In the absence of any other confirmatory evidence the reaction should be repeated. A diagnosis should not be made on the result of a single serum test of this type. If the repeated test records a similar result, it can be taken that the patient is suffering from gonorrhœa.

(ii.) Sub-acute cases in which the infection is limited to the anterior urethra.

(iii.) Old standing cases in which, owing to effective treatment, the case is approaching final cure.

$A$ negative reaction (-) obtained when gonococci are exhibited in the secretions should be regarded as :-

(i.) An early acute infection of less than twenty-one days' duration.

(ii.) An infection, limited in the male to the anterior urethra, or in the female to the lower genitalia.

(iii.) An infection of the seminal vesicles or prostate, which has been treated by massage so that drainage has been established and the absorption of toxic material stopped. It should be clearly understood that a single negative serum test in such circumstances is no criterion of cure.

$A$ negative reaction (-) obtained when no gonococci are demonstrable in smears or cultures indicates that:264 


\section{FIXATION TEST FOR GONORRHEA}

(i.) The case is not a gonococcal infection; or

(ii.) The case is cured.

At this stage the value of the reaction in a test of cure may be discussed. The reader will have realised that a single negative serum test by itself is insufficient evidence on which to base a standard of cure, but when compared with the other means of testing at our disposal, such as smears and cultures, it holds pride of place. A negative result shows that absorption of toxin has ceased, but this, although of great importance, is not conclusive evidence that the gonococci have been expelled from the tissues. Therefore, in a test of cure, all evidence, both clinical and pathological, must be considered. It is suggested that, if the patient shows no clinical signs and pathological evidence of the disease (serum tests, smears, cultures, etc.) is negative, normal life (with the single exception of coitus) should be allowed for one month. Then the patient should be re-examined and all tests repeated. If these still yield negative results a further month of normal life should be allowed before the final tests are made. When these are satisfactory, the patient may be regarded as cured. Throughout a test of cure the following points should be remembered.

(i.) It is certain that a patient with a persistently positive complement fixation test is still infectious. It is therefore useless to perform a test of cure until the complement fixation test for gonorrhœa has become negative.

(ii.) No patient should be discharged as cured on the strength of a single negative serum test. This is insufficient evidence on which to assume that the infection has been eradicated.

(iii.) The importance of vesicular smears and especially cultures as a complementary investigation to a negative complement fixation test in a routine test of cure in the male cannot be over-estimated. I have found that in cases of chronic gonococcal vesiculitis which have been treated by massage and where the complement fixation reaction for gonorrhœa has been reduced from positive to negative, gonococci may still be grown in culture by a special method which I have devised from the vesiculo-prostatic material in 22 per cent. of cases. 


\section{BRITISH JOURNAL OF VENEREAL DISEASES}

(iv.) If a case is prematurely discharged as cured on the strength of a single negative complement fixation test and gonococci are still present in the tissues, the serum test will generally become positive again after an interval of about one month.

To sum up, in view of what has been stated in the foregoing pages, it will be seen that in order to interpret correctly the result of a complement fixation test for gonorrhœa it is essential to understand the significance of an "open" or " closed" infection. Either type of infection (or a modification thereof) is present at any stage of the disease, and it may be surmised from the result of a serum reaction whether or not drainage is established. Thus a strongly positive reaction is indicative of a "closed" infection with little or no drainage and considerable absorption of the toxins of the infection. On the other hand, a negative result in a known case of gonorrhœa is evidence of excellent drainage and little or no absorption of toxin. Between these two extremes varying stages of drainage and absorption of the toxic material occur, and the result of a serum reaction at any time during the disease is dependent on whichever of these factors are present at the time of the test. If these facts be borne in mind, there will be little difficulty in correlating the result of a serum test with the clinical picture. Two exceptions to the above general rule occur :-

(i.) Patients ( $0 \cdot 1$ per cent.) who appear to lack the necessary tissue reaction to form antibodies to the gonococcus; and

(ii.) Patients who have been treated with vaccine.

A negative complement fixation test, therefore, does not of necessity exclude a gonococcal infection, but must be interpreted in the light of the clinical condition of the patient together with any other pathological findings (smears, cultures) which are available.

On the other hand, providing no vaccine has been administered, a positive test is always indicative of the presence of living gonococci in the tissues. It has frequently been stated that a positive complement fixation test for gonorrhœa may take some time after the patient has been cured, to become negative. In my opinion this 266 


\section{FIXATION TEST FOR GONORRHEA}

is not the case. If a positive serum reaction is obtained when the patient is apparently cured, further careful search will, as a rule, reveal a focus of infection. In the male this is usually found in the seminal vesicles.

A positive complement fixation test due to the reaction of the tissues against the presence of vaccine will not persist for more than 6-7 weeks after cessation of the vaccine administration.

Early acute untreated cases of gonorrhœa may be expected to give a positive serum test from the first week onwards of exposure to infection.

Chronic cases of gonorrhœa which have been insufficiently treated usually give a positive result. The seat of the infection in the male appears to be in the seminal vesicles which may act as a reservoir of infection, whilst in the female the uterus or higher adnexa is usually involved.

Chronic cases of gonorrhœa which have been vigorously treated so that efficient drainage is established may give a negative result; but, if in such a case treatment is prematurely stopped on the strength of one negative complement fixation test, a positive result is usually recorded after an interval of one month.

The test is essential to a " test of cure," but, if a negative result is recorded, it should be supported by smears and cultures. It cannot be too strongly emphasised that no patient should be discharged on the strength of a single negative complement fixation test.

The test should be used to estimate the efficiency of antibody formation as a result of vaccine treatment.

Finally, there is little evidence to show that crossfixation with the antibodies of other gram-negative cocci would confuse the issue either in diagnosis or in a test of cure.

\section{REFERENCES}

(I) Price, I. N. O. : "The Complement Fixation Test for Gonorrhœa." London County Council Publication No. 2995.

(2) ARkwright, J. A. : Journ. of Hygiene, I9I2, II, No. 4, 5I5.

(3) Oliver, J. O.: Journ. of Hygiene, I928, 29, No. 3, 259.

(4) PrICE, I. N. O. : British Med. Journ., I93I, April 4th, 579.

(5) Mascall, W. N. : British Med. Journ., I93I, October 3rd, 607. 